\title{
Comparative Study of the Birefringence in Photonic Crystal Fiber Lasers
}

\author{
N. Rouchdi', M. Abouricha1 ${ }^{*}$, A. Boulezhar ${ }^{1}$, M. Kriraa $^{2}$ \\ ${ }^{1}$ Laboratory of theoretical and Applied Physics, Faculty of Sciences-Ain Chok, Hassan II Casablanca University, \\ Casablanca, Morocco \\ ${ }^{2}$ Thermal Group LPMMAT, Department of Physics, Faculty of Sciences-Ain Chok, Hassan II Casablanca \\ University, Casablanca, Morocco \\ Email: "mmabouricha@gmail.com
}

Received 27 June 2015; accepted 17 November 2015; published 20 November 2015

Copyright (C) 2015 by authors and Scientific Research Publishing Inc.

This work is licensed under the Creative Commons Attribution International License (CC BY).

http://creativecommons.org/licenses/by/4.0/

\section{cC) (i) Open Access}

\begin{abstract}
In this paper, we study the birefringence in photonic crystal fiber lasers PCFs and in conventional fiber lasers in the bi-directional pump scheme in the linear cavity laser. We show that the value of birefringence in photonic crystal fibers is smaller than that of conventional fiber lasers [1].
\end{abstract}

\section{Keywords}

Pump Schemes, PCF Lasers, Fiber Lasers, Yb-Doped Fiber Lasers, Thermal Effects in Fiber Lasers

\section{Introduction}

Photonic crystal fibers (PCFs) are attracting increasing interests because of their unique properties such as: endlessly single-mode guiding, freedom of dispersion characteristics, and large mode area [2] [3]. In this work, we focus on PCFs in which a core doped with $\mathrm{Yb}^{3+}$ is surrounded by a lower index cladding, which is, surrounded by an air-clad region, in turn, surrounded by a second lower index cladding index.

We use the rate equation for finding the expressions of the temperature dependence in Regions I, II, III, and IV [4] [5]. In order to get this dependence we utilize the results of the expressions for the stress components ( $\sigma_{r}(r), \sigma_{\varphi}(r)$, and $\sigma_{y}(r)$ ), the change in the index of refraction and therefore the birefringence with the result of the birefringence in Regions I, II, III, and IV. Then, the obtained results are compared for different pump schemes for giving the design guidelines to ensure maximum heat dissipation and pump powers.

\section{Theoretical Model}

Figure 1 displays a schematic illustration of Yb doped PCFs [6]. For the convenience of analysis, the pump

"Corresponding author.

How to cite this paper: Rouchdi, N., Abouricha, M., Boulezhar, A. and Kriraa, M. (2015) Comparative Study of the Birefringence in Photonic Crystal Fiber Lasers. Optics and Photonics Journal, 5, 320-325.

http://dx.doi.org/10.4236/opj.2015.511030 


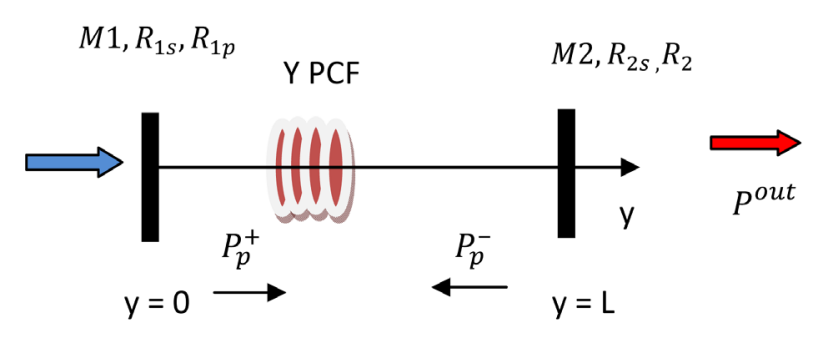

Figure 1. Schematic illustration of a linear cavity [7].

light and output laser can be expressed as follows:

$$
\begin{aligned}
& \pm \frac{P_{p}^{ \pm}(y)}{\partial y}=\Gamma_{p}\left[\left(\sigma_{a p}+\sigma_{e p}\right) N_{b}(x)-\sigma_{a p} N_{y t}\right] \times P_{p}^{ \pm}(y)-\alpha_{p} P_{p}^{ \pm}(y) \\
& \pm \frac{P_{s}^{ \pm}(y)}{\partial y}=-\Gamma_{s}\left[\left(\sigma_{a s}+\sigma_{e s}\right) N_{b}(y)-\sigma_{a s} N_{y t}\right] \times P_{s}^{ \pm}(y)-\alpha_{s} P_{s}^{ \pm}(y) \\
\frac{N_{b}(y)}{\partial t}= & \left(\frac{\lambda_{p} \Gamma_{p} \sigma_{a p}}{h c A_{c o}}\right)\left[N_{y t}-N_{b}(y)\right]\left[P_{p}^{+}(y)+P_{p}^{-}(y)\right]-\left(\frac{\lambda_{p} \Gamma_{p} \sigma_{a p}}{h c A_{c o}}\right)\left[N_{y t}-N_{b}(y)\right] \\
& -\left(\frac{\lambda_{p} \Gamma_{p} \sigma_{a p}}{h c A_{c o}}\right)\left[N_{y t}-N_{b}(y)\right]\left[N_{y t}-N_{b}(y)\right]\left[P_{p}^{+}(x, t)+P_{p}^{-}(, y)\right] \\
& -\left(\frac{\lambda_{p} \Gamma_{p} \sigma_{a p}}{h c A_{c o}}\right)\left[N_{y t}-N_{b}(y)\right]\left[P_{p}^{+}(x)+P_{p}^{-}(y)\right]-\frac{N_{b}(y)}{\tau}
\end{aligned}
$$

where $N_{b}(y)$ is the upper laser level population density, $N_{y t}$ is the concentration of $\mathrm{Yb}^{3+}, P_{p}^{-}(, y)$ and $P_{p}^{-}(, y)$ represent the power of forward and backward propagation pump light, respectively. $P_{s}^{-}(, y)$ and $P_{s}^{-}(, y)$ represent the power of forward and backward propagation laser power, respectively, $\sigma_{a s}$ and $\sigma_{e s}$ are the laser absorption and emission cross section, $\sigma_{a p}$ and $\sigma_{e p}$ are the pump light absorption and emission cross-section respectively, $\Gamma_{p}$ and $\Gamma_{s}$ are the power-filling factors [7].

Figure 2 shows the injection mode in fiber laser PCFs. and the radial coordinate $r$ and the tangential $\varphi$. The quantities $a, b, c$ and $e$ are the radius of the core, the inner cladding, the air-clad and the outer cladding, respectively. The temperature distribution in a fiber reported in Ref. [4] is necessary for determining the radially varying index of refraction $\mathrm{d} n / \mathrm{d} T$, and the calculate stresses. By using Equations (1)-(3), Abouricha et al. [4] demonstrated that the temperature expressions of the stress components can be written as: [1] [8]

$$
\begin{gathered}
\sigma_{r}(r)=\frac{\alpha E}{(1-\vartheta)}\left[\frac{1}{b^{2}} \int_{0}^{e} T(r) r \mathrm{~d} r-\frac{1}{r^{2}} \int_{0}^{r} T(r) r \mathrm{~d} r\right] \\
\sigma_{\varphi}(r)=\frac{\alpha E}{(1-\vartheta)}\left[\frac{1}{b^{2}} \int_{0}^{e} T(r) r \mathrm{~d} r-\frac{1}{r^{2}} \int_{0}^{r} T(r) r \mathrm{~d} r-T(r)\right]
\end{gathered}
$$

In the case where the fiber end faces are free of traction

$$
\sigma_{y}(r)=\sigma_{r}(r)+\sigma_{\varphi}(r)
$$

where $\sigma_{r}(r), \sigma_{\varphi}(r)$, and $\sigma_{y}(r)$ are of the radial, tangential, and azimuth, stress components, respectively, and $\alpha$, Eand $v$ are the thermal expansion coefficient, Young's modulus, and Poisson's ratio, respectively.

The length of optical fibers is much greater than that of a typical fiber outside radius (b). Thus, we can invoke the plane-strain approximation [9] in which the y strain component everywhere. We also can define the birefringence $\Delta n_{B}^{I, I I, I I I, I V}(r)$, given by.

$$
\Delta n_{B}^{I, I, I I I, I V}(r)=\Delta n_{r}^{I, I I, I I I, I V}(r)-\Delta n_{\varphi}^{I, I I, I I I, I V}(r)
$$

Therefore, the expressions in different regions of the fiber can be given by [10]: 


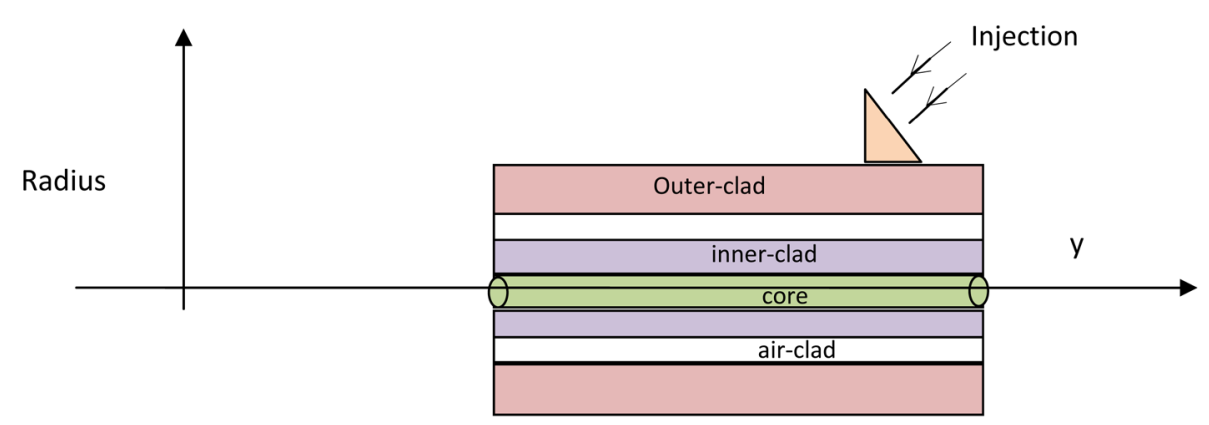

Figure 2. Transversal injection of the PCFs.

$$
\begin{gathered}
\Delta n_{B}^{I}(r)=\frac{n_{0}^{3}}{2} B\left(\sigma_{r}^{I}(r)-\sigma_{\varphi}^{I}(r)\right) \\
\Delta n_{B}^{I I}(r)=\frac{n_{0}^{3}}{2} B\left(\sigma_{r}^{I I}(r)-\sigma_{\varphi}^{I I}(r)\right) \\
\Delta n_{B}^{I I I}(r)=\frac{n_{0}^{3}}{2} B\left(\sigma_{r}^{I I I}(r)-\sigma_{\varphi}^{I I I}(r)\right) \\
\Delta n_{B}^{I V}(r)=\frac{n_{0}^{3}}{2} B\left(\sigma_{r}^{I V}(r)-\sigma_{\varphi}^{I V}(r)\right) \\
B=B_{\|}-B_{\perp}
\end{gathered}
$$

where $B_{\|}$and $B_{\perp}$ are the parallel and perpendicular stress-optic coefficients. Their values are $4.5 \times 10^{-8} \mathrm{~cm}^{2} / \mathrm{kg}$, $27.7 \times 10^{-8} \mathrm{~cm}^{2} / \mathrm{kg}$ respectively [1]. The numerical values of $E$ and $v$ are $E=73 \mathrm{GPa}=7.45 \mathrm{~kg} / \mathrm{cm}^{2}$ and $v=0.16$ [5].

Equations (8)-(11) show that the fiber birefringence depends only on the thermally induced stresses.

Using the expressions of the temperature dependence in Regions I, II, III, and VI [4], and Equations (8)-(11) we calculate the birefringence in different regions of cavity laser

\section{Simulation and Discussion}

For the purposes of illustration, unless indicated otherwise, the parameters used in the simulation are $\lambda_{p}=975$ $\mathrm{nm}, \lambda_{s}=1080 \mathrm{~nm}, R_{1 s}=0.98, R_{2 s}=0.04, L=5 \mathrm{~m}, \tau=0.8 \mathrm{~ms}, \sigma_{a p}=2 \times 10^{-24} \mathrm{~m}^{2}, \sigma_{e p}=2 \times 10^{-24} \mathrm{~m}^{2}$, $\sigma_{a s}=3.1 \times 10^{-27} \mathrm{~m}^{2}, \quad \sigma_{e s}=4.2 \times 10^{-25} \mathrm{~m}^{2}, \quad N_{y t}=1.6 \times 10^{26}, \quad \Gamma_{p}=0.0012, \quad \Gamma_{s}=0.8, \quad \alpha_{s}=5 \times 10^{-3} \mathrm{~m}^{-1}$, $\alpha_{p}=3.1 \times 10^{-3} \mathrm{~m}^{-1}$ fiber core diameter $D=10 \mu \mathrm{m}$ and $N_{A}=0.05$.

The boundary conditions for the laser propagation equation can be written as:

$$
\begin{gathered}
P_{s}^{+}(0)=R_{1 s} P_{s}^{-}(0) \\
P_{s}^{-}(L)=R_{2 s} P_{s}^{+}(L)
\end{gathered}
$$

In addition, for the pump:

In the bidirectional pump scheme:

$$
P(0)=100 \mathrm{~W} \text { and } P(L)=100 \mathrm{~W}
$$

In the forward pump scheme:

$$
P(0)=200 \mathrm{~W} \text { and } P(L)=0
$$

Using analytical and numerical calculations, the finite-difference method (FDM) and a simple model of (PCFs), we have determined the distributions of pump and laser along the PCFs in the forward and bidirectional pumped YPCFs, and the birefringence in PCF laser dope ytterbium in cases the forward and bi-directional pump scheme respectively as function of the radius. 
Figure 3 and Figure 4 show the distributions of pump and laser along the PCFs in the forward and bidirectional pumped YPCFs. All the launched pump powers are the same: $200 \mathrm{~W}$. We notice an increase of the speed of forward signal power along the PCFs in both cases (the forward and bidirectional pump schemes), and it can be seen that this increase slows down along the YPCFs for the forward pumped lasers. As a result, the forward pump power and inverse population correspondingly decrease. The bidirectional pump scheme helps to the equivalently distribution of the pump power, avoiding the optical and thermal damages.

Both Figure 5 and Figure 6 show the birefringence as function of the radius in PCF lasers doped ytterbium in the bidirectional pump scheme and forward pump scheme respectively. Their values do not have a great effect on the quality of laser beam in these pump schemes. However, the birefringence in bidirectional pump scheme is diminishing their values in the forward pump scheme. Therefore, the second advantage of the bidirectional pump scheme is to have a high quality of laser beam then the forward pump scheme and then in conventional fiber lasers [1].

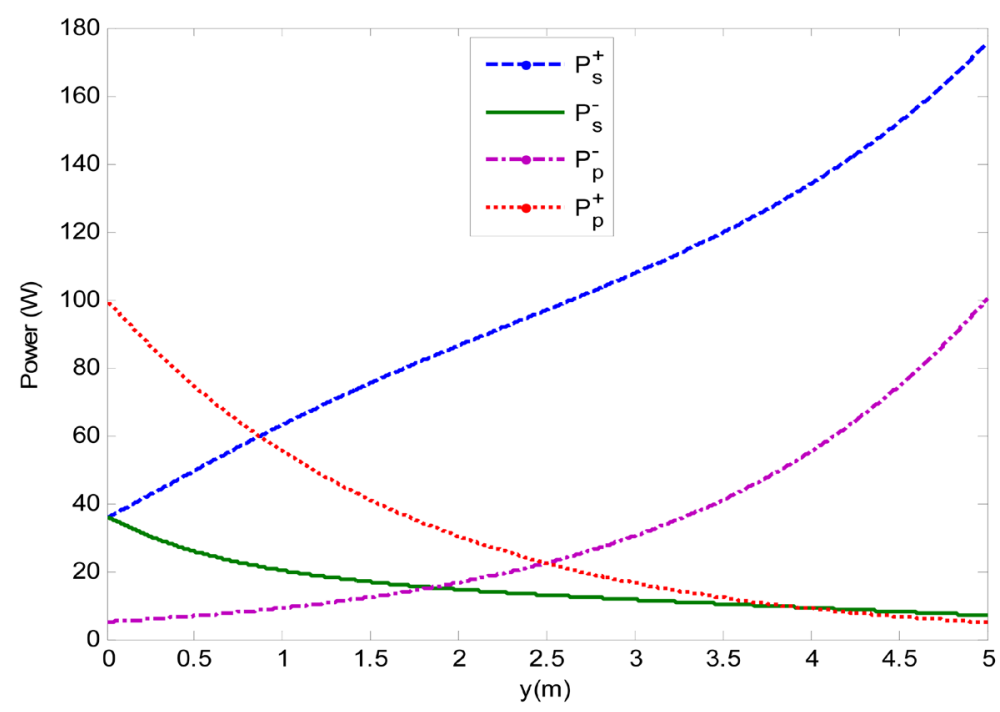

Figure 3. Distributions of pump and laser powers along the PCFs in the bidirectional pump scheme.

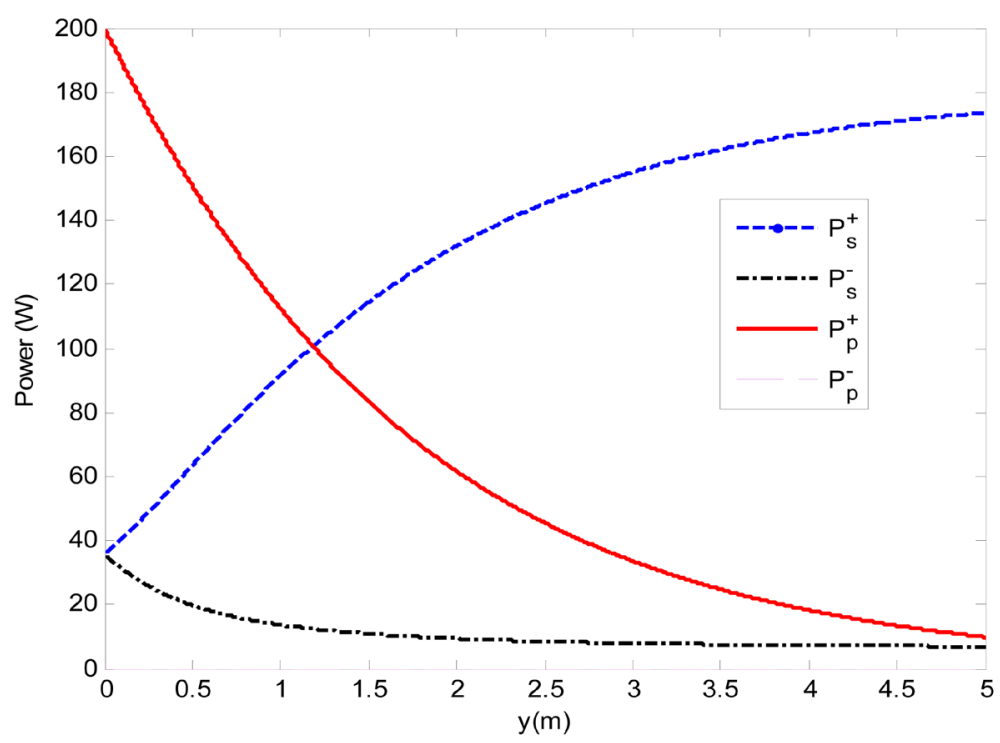

Figure 4. Distributions of pump and laser powers along the PCFs in the forward pump scheme. 


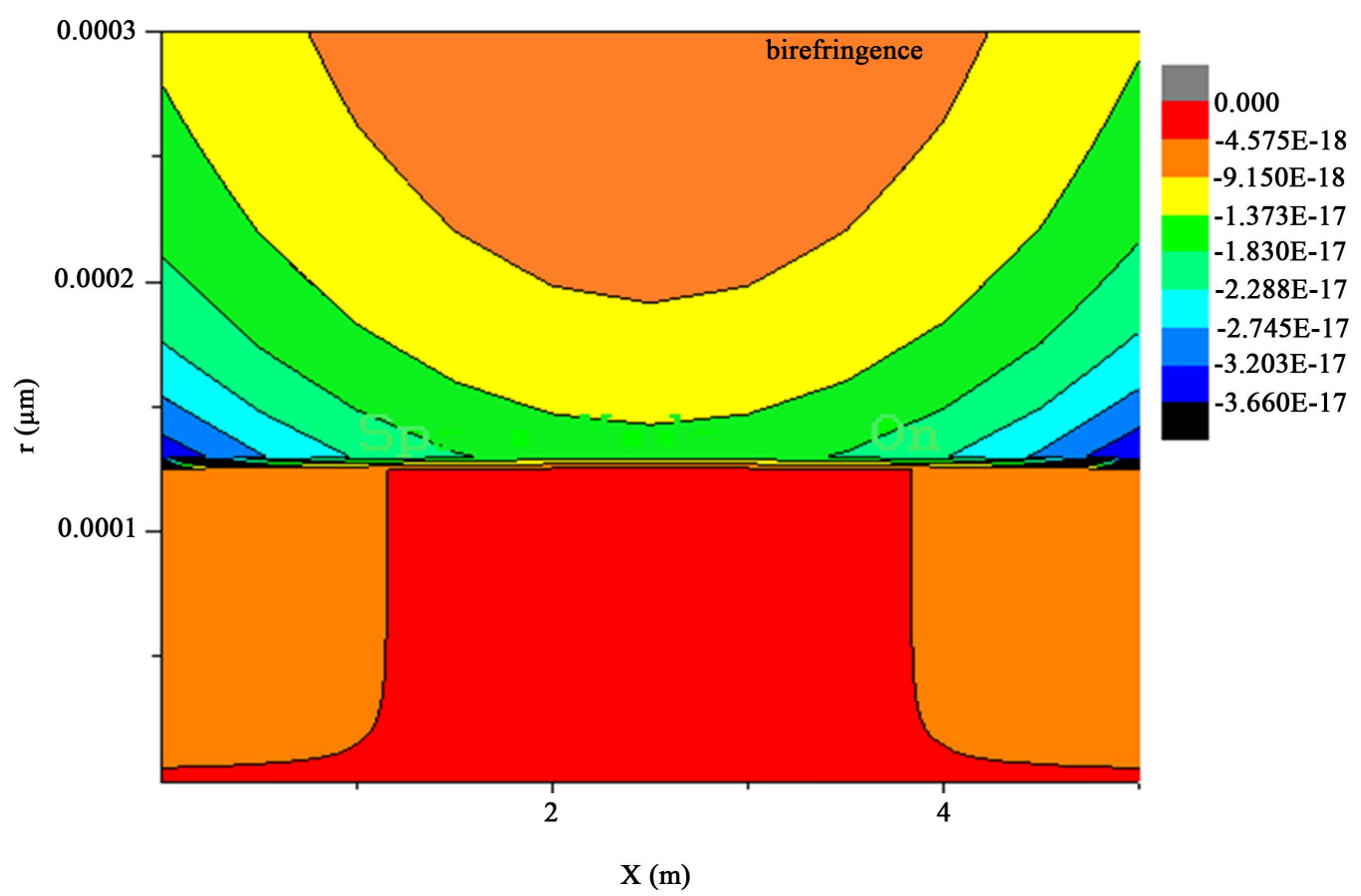

Figure 5. Birefringence in PCF lasers doped by ytterbium in case of the bi-directional pump scheme.

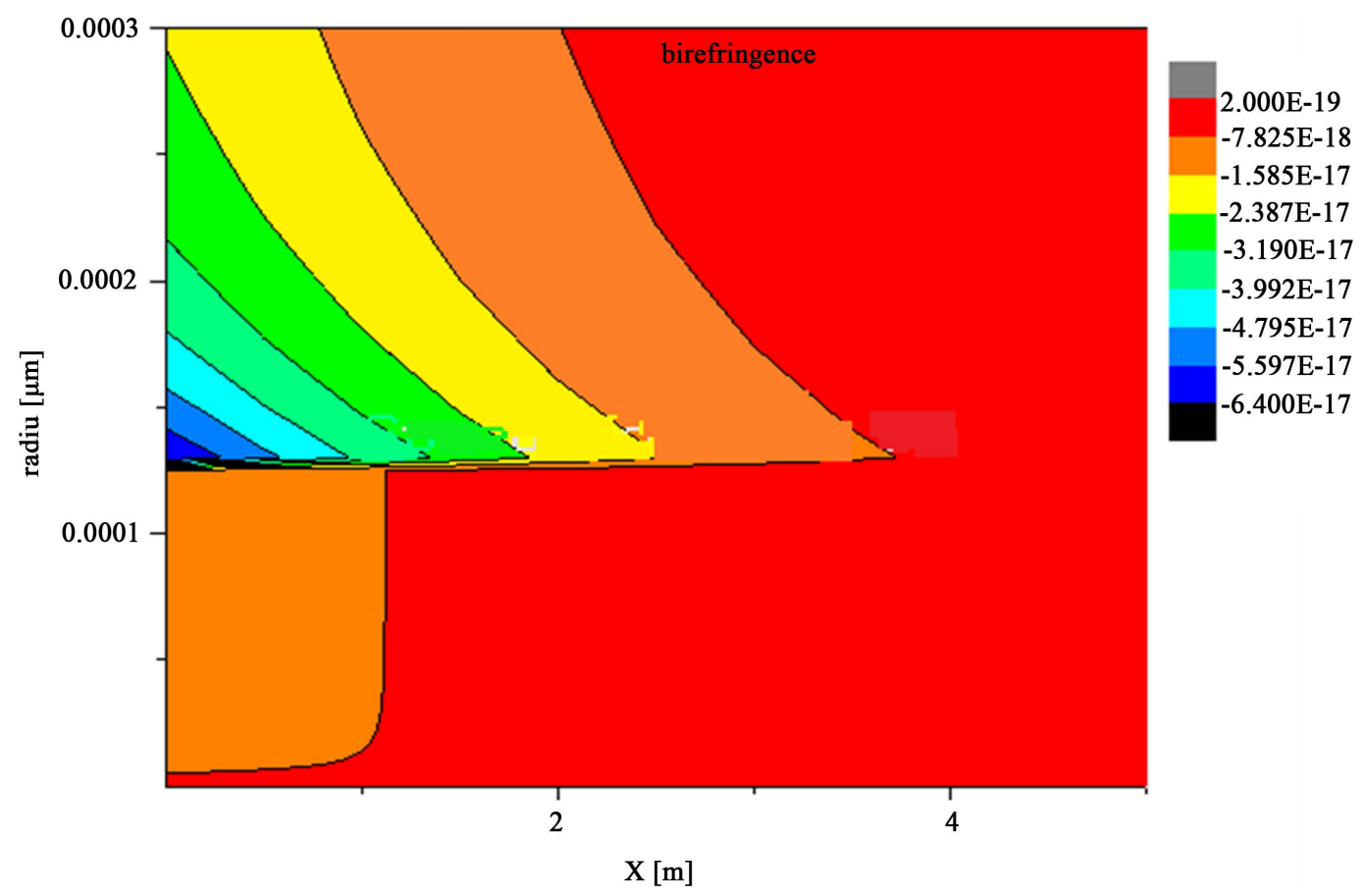

Figure 6. Birefringence in PCF lasers doped ytterbium in case of the forward pump scheme.

\section{Conclusion}

In this paper, we have compared the birefringence in photonic crystal fibers (PCFs) with the birefringence in conventional fibers.

In summary, regarding the birefringence, their value in PCFs are less than that found in conventional fiber 
lasers [1] and do not have a great effect on the quality of the laser beam in different pump schemes, especially in the bi-directional pumping. Hence, after this comparison, we optimized the x position of the transversal pump in the laser cavity (PCFs) which was the most convenient in specific conditions.

\section{References}

[1] Brown, D.C. and Hoffman, H.J. (2001) Thermal, Stress, and Thermo-Optic Effects in High Average Power DoubleClad Silica Fiber Lasers. IEEE Journal of Quantum Electronics, 37, 207-217.

[2] Knight, J.C. and Russell, P.St.J. (2002) New Ways to Guide Light. Science, 296, 276-277. http://dx.doi.org/10.1126/science.1070033

[3] Knight, J.C., Briks, T.A., Cregan, R.F., et al. (1998) Large Mode Area Photonic Crystal Fiber. Electronics Letters, 34, 1347-1349. http://dx.doi.org/10.1049/el:19980965

[4] Abouricha, M., Boulezhar, A. and Habiballah, N. (2013) The Comparative Study of the Temperature Distribution of Fiber Laser with Different Pump Schemes. Open Journal of Metal, 3, 64-71. http://dx.doi.org/10.4236/ojmetal.2013.34010

[5] Bass, M., Ed. (1995) Handbook of Optics (Sponsored by the Optical Society of America), Vol. II, Devices, Measurements, and Properties. McGraw-Hill, New York.

[6] Brown, D.C. (1998) Nonlinear Thermal Distortion in YAG Rod Amplifiers. IEEE Journal of Quantum Electronics, 34, 2383-2392. http://dx.doi.org/10.1109/3.736113

[7] Limpert, J., Schreiber, T., Liem, A., Nolte, S., Zellmer, H., Peschel, T., Guyenot, V. and Tünnermann, A. (2003) Thermo-Optical Properties of Air-Clad Photonic Crystal Fiber Lasers in High Power Operation. Optic Express, 11, 2984.

[8] Eggleston, J.M., Kane, T.J., Kuhn, K., Unternahrer, J. and Byer, R.L. (1984) The Slab Geometry Laser-Part I: Theory. IEEE Journal of Quantum Electronics, 20, 289-301. http://dx.doi.org/10.1109/JQE.1984.1072386

[9] Boley, B.A. and Weiner, J.H. (1985) Theory of Thermal Stresses. Reprint Edition, Kreiger, Melbourne.

[10] Abouricha, M., Boulezhar, A., El Mouden, M. and Kriraa, M. (2014) Theoretical and Numerical Study of Stress and Thermo-Optic in Photonic Crystal Fiber Laser in Different Pump Schemes. Open Journal of Metal, 4, 120-130. http://dx.doi.org/10.4236/ojmetal.2014.44014 